\title{
Fully automatic segmentation of intima/adventitia of the vessel using Bezier curve from intravascular ultrasound
}

\author{
Kwang Baek Kim¹, Doo Heon Song ${ }^{2}$ \\ ${ }^{1}$ Division of Computer Software Engineering, Silla University, Busan, Korea \\ ${ }^{2}$ Department of Computer Games, Yong-In SongDam College, Yong-in, Korea
}

\begin{tabular}{|c|c|}
\hline Article Info & ABSTRACT \\
\hline Article history: & Although medical image segmentation field is regarded as one of most \\
\hline Received Jul 31, 2020 & $\begin{array}{l}\text { established fields, still fully automatic segmentation to extract target object } \\
\text { with high accuracy from intravascular ultrasound (IVUS) is very active area }\end{array}$ \\
\hline Revised Sep 22, 2020 & of research. In this paper, we propose a fully automatic morphological approach \\
\hline Accepted Oct 15, 2020 & $\begin{array}{l}\text { using Bezier curve in interpolating the boundaries of intima/adventitia of the } \\
\text { vessel from IVUS with careful binarization algorithms. In experiment with } 800\end{array}$ \\
\hline Keywords: & $\begin{array}{l}\text { IVUS images, the proposed method is as good as fuzzy C-means based } \\
\text { approach in comparison with human expert's result with } 84.4 \% \text { satisfaction }\end{array}$ \\
\hline $\begin{array}{l}\text { Adventitia } \\
\text { Automatic segmentation }\end{array}$ & $\begin{array}{l}\text { and better than other morphological method in all performance indices of } \\
\text { curve fitting with } 97.02 \% \text { in accuracy and } 58.19 \% \text { in precision. }\end{array}$ \\
\hline
\end{tabular}

Bezier curve

Intima

IVUS

This is an open access article under the CC BY-SA license.

\section{Corresponding Author:}

Kwang Baek Kim

Division of Computer Software Engineering

Silla University

Busan 46958, Korea

Email: gbkim@silla.ac.kr

\section{INTRODUCTION}

Intravascular ultrasound (IVUS) is an intra-operative imaging modality that facilitates observing and appraising the vessel wall structure of the human coronary arteries. IVUS is regularly used to locate the atherosclerosis lesions in the coronary arteries to study the lumen and plaque dimensions, and to guide intervention and stent deployment [1]. Segmentation of arterial wall boundaries from the IVUS images is not only crucial for quantitative analysis of the vessel walls and plaque characteristics but is also necessary for generating 3D reconstructed models of the artery [2]. In particular, delineating the interior (lumen) and exterior (media) vessel walls is problematic due to the presence of various artifacts such as motion of the catheter after a heart contraction, guide wire effects, bifurcation and side branches or similar echogenicity between the vessel wall and some plaques. In some cases, even the differences in transducer frequencies affect the segmentation results [3]. Automatic detection of the vessel wall (media-adventitia) and borders of lumen that is at the innermost surface of atherosclerotic plaques is required to quantify the degree of stenosis and measure the luminal area in which blood flows. Such procedure is a basic step for the evaluation of the morphology of the vessel under analysis and the detection of possible atherosclerotic lesions [4, 5].

There have been many different approaches to find an effective segmentation result of intimaadventitia of vessel in this problem domain. Deformable models have been used in [6-8] to detect the border of lumen/intima and media/adventitia. However, every first frame of the sequence needs user interactions to manually segment the lumen and media in this approach. Edge-tracking and gradient-based techniques are typically semiautomatic and require a precise initialization. They also typically rely on an energy 
minimization (optimization) framework to achieve an accurate segmentation [9, 10] but classic active contours typically do not perform well on IVUS images [11].

There are several neural network-based approaches to this problem $[12,13]$. A carefully controlled neural network showed competent performance with human expert [12]. A sequential forward selection process using support vector machine (SVM) has been employed to conduct an in-depth analysis of several image features in [13]. A limitation of their analysis is that it only works for artifact-free IVUS sequences. As we can see, most approaches have employed either a type of energy minimization method or require annotated data to train the neural network classifier.

There are also several interesting approaches using fuzzy algorithms as combined with neural network [14] or as a fuzzy C-means (FCM) based clustering mechanism [4, 15]. In [14], the lumen is segmented by a combination of image gradient and fuzzy connectedness model and the media/adventitia border is extracted by a fast-marching model. An interesting combinatory approach proposed in [16] used gray-level co-occurrence matrix (GLCM) to define features of Intima and use FCM to extract adventitia. FCM is usually efficient to extract target object from ultrasound images but it may suffer from hard initialization of number of clusters as is thus some kind of dynamic control might be necessary [17].

In such a combined approach between FCM and morphological algorithms like [17], we need a high quality, well contrasted IVUS images since there are many different types of artifacts that we should exclude in the process [3]. There have been studies to obtain better quality images by efficient binarization techniques [18-20] that should be done before main fuzzy controlled algorithms are applied.

In this paper, we propose an efficient morphological method to extract intima/adventitia without FCM. Instead, we apply Bezier curve in extracting adventitia. Then, based on extracted adventitia, we apply convex parabola to enhance the contrast for Intima area and apply binarization algorithm to extract Intima. The Bezier curve is popular in computer graphics and computer-aided application as well as medical image processing [21-25]. The Bezier curves were used to detection and interpolation of the salient points for approximating the boundaries of the lung fields or prostate region in those researches. Inherently, the Bezier curve is characterized by convex hull and the curve is contained inside control polygon, guaranteeing that the generated curve will not derail off its control polygon [26]. After automatic segmentation by proposed method, we compute the area of intima/adventitia that would be used for medical doctors in decision making process.

\section{METHOD}

The overall assumption we have for IVUS image in this research is as;

- Assumption (1): Boundaries of adventitia has relatively higher intensity values than those of intima [16].

- Assumption (2): Adventitia and intima area always contain the center of the image.

\subsection{Extracting adventitia}

Firstly, we apply appropriate binarization algorithm [20] to extract the candidate area of adventitia under assumption (1). Then the proposed algorithm searches for appropriate control point from the center of image (under assumption (2)) to increase the searching angle gradually. Bezier curve has parameterized equation for representing the curve and use multiple control point to interpolate the shape of the model based on Bernstein basis function. We can define the (n-1)-th Bezier curve recursively as a closed form given $\mathrm{n}$ control points by using De Casteljau algorithm as shown in (1) and (2) [27].

$$
\begin{aligned}
& B(t)=\sum_{i=0}^{n} P_{i} b_{1-n}(t), t \in[0,1\rceil \\
& b_{i, n}(t)={ }_{n} C_{\bar{l}} t^{\bar{l}}(1-t)^{n-1}, i=0, \ldots, n
\end{aligned}
$$

where $B(t)$ is a Bezier curve with n control points $P_{i}$ with time $t$. Figure 1 shows an example of Bezier curve with $t=0.25$ and how the curve is interpolated by multiple control points. Then, the adventitia of the vessel is modeled as connecting and expanding Bezier curve as shown in Figure 2.

\subsection{Extracting intima}

Then, the next step is extracting Intima. In order to enhance the intensity contrast between adventitia/intima boundary lines and other areas in the region of interest (ROI) that contains already extracted adventitia by method explained in section 2.1, we apply convex parabola equation as shown in (3). 


$$
f(x)=255\left(\frac{x}{127}-1\right)^{2}
$$

The applied convex parabola equation transforms the given intensity value to emphasize the highest and lowest values and deemphasize the neutral intensity as shown in Figure 3. And the effect of such treatment is shown as Figure 4 (b). The final extraction of Intima needs binarization procedure and labeling procedure to form the object model and the result is as shown in Figure 4(c). Since all given IVUS images have the same size in that 2,500 pixels are within $1 \mathrm{~mm}^{2}$ area thus we can compute the area within the adventitia and Intima of the vessel as counting pixels inside the area (the variable ' $x$ ' of the (4)) under (4). The result or the proposed method displays both Adventitia and Intima area as shown in Figure 5.

$$
f(x)=\frac{x}{2500}
$$

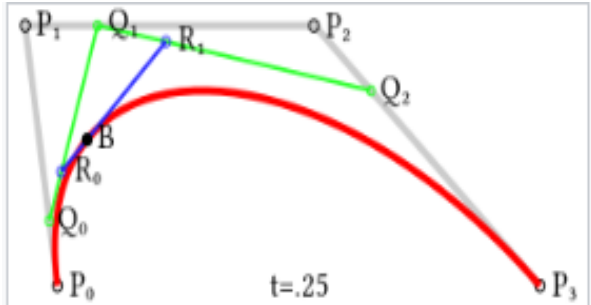

Figure 1. Bezier curve example

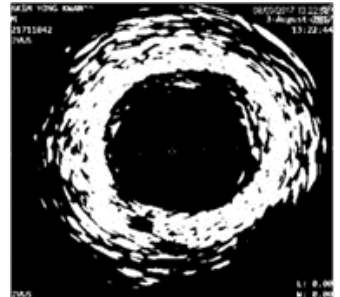

(a)

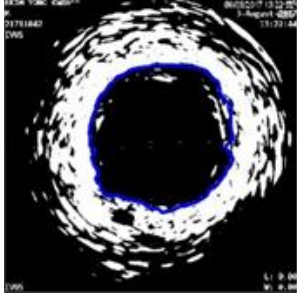

(b)

Figure 2. Extracting adventitia; (a) binarized IVUS input, (b) extracted adventitia

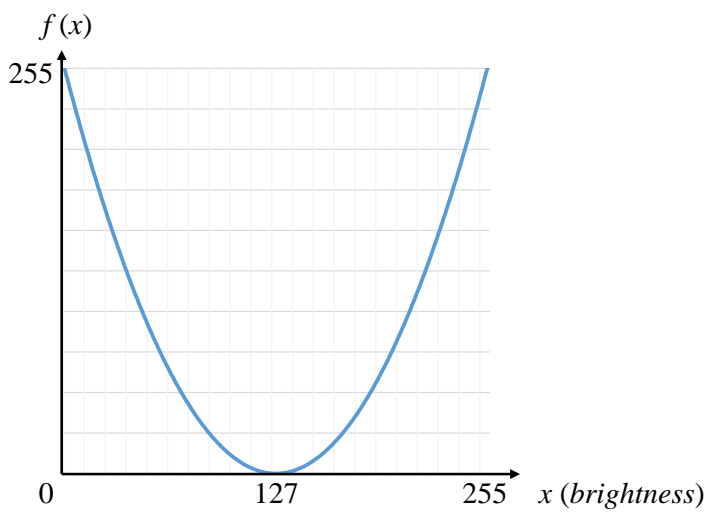

Figure 3. Transform of intensity be convex parabola function

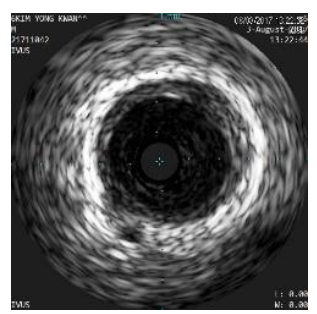

(a)

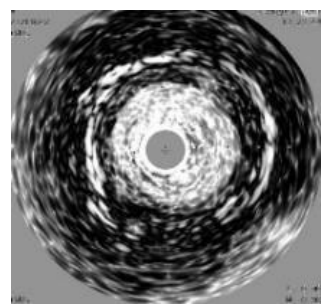

(b)

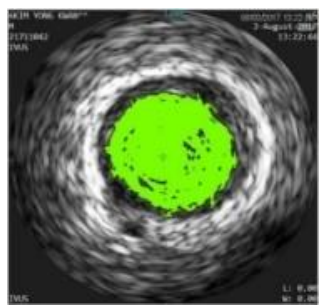

(c)

Figure 4. Extraction of intima; (a) input image, (b) convex parabola effect, (c) intima extracted 


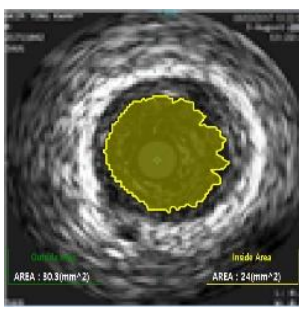

(a)

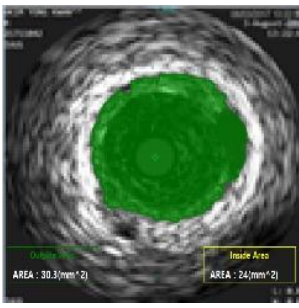

(b)

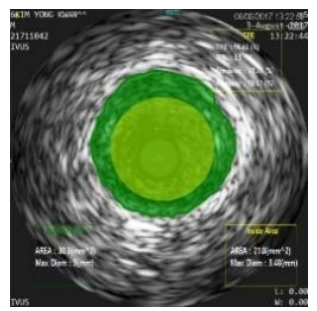

(c)

Figure 5. Adventitia/intima display by the proposed method; (a) intima, (b) adventitia, (c) combined

\section{RESULT}

The proposed method is implemented using visual studio 2017 C\# with Intel® Core ${ }^{\mathrm{TM}}$ i5-6200 CPU @ $2.50 \mathrm{GHz}$ and 8 GB RAM with 800 IVUS images obtained from Baek Hospital located in Busan, Korea. We demonstrate several examples of intima/adventitia extractions by proposed method as shown in Figure 6. To evaluate the performance of the proposed method, we take pathologists' manual examination result as the gold standard and see how much the system's discrepancy is in area as shown in Table 1.

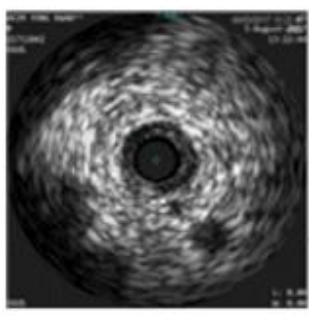

(a)

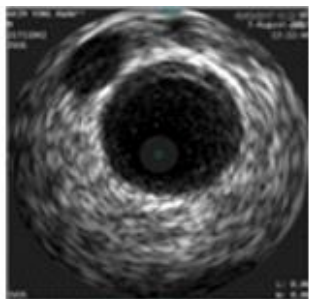

(c)

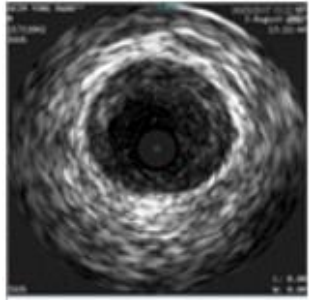

(e)

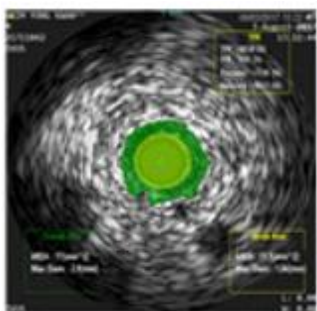

(b)

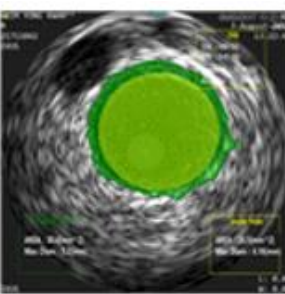

(d)

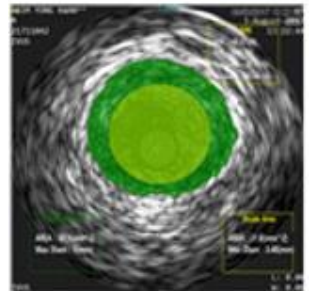

(f)

Figure 6. Examples of adventitia/intima extractions by proposed method; a) input, (b) extracted, (c) input, (d) extracted, (e) input, (f) extracted

Table 1. Area discrepancy by proposed method in $\mathrm{mm}^{2}$

\begin{tabular}{ccc}
\hline Discrepancy & \# of Images & Rate \\
\hline$<15$ & 602 & $75.3 \%$ \\
$15 \sim 20$ & 32 & $4.0 \%$ \\
$20 \sim 25$ & 41 & $5.1 \%$ \\
$>25$ & 125 & $15.6 \%$ \\
Total & 800 & $100.0 \%$ \\
\hline
\end{tabular}


Field experts evaluated the system's performance as 'excellent' in $75.3 \%$ of the cases $\left(<15 \mathrm{~mm}^{2}\right.$ discrepancy) but not satisfied with cases having over $25 \mathrm{~mm}^{2}$ thus the satisfaction rate would be $84.4 \%$ and that is similar to the best reported parameterized cases of FCM of 86\% [4]. Another performance measure we take is the actual discrepancy rate from human expert's decision with true positive rate and false positive rate as well as precision and accuracy that are defined as (5) to (8).

$$
\begin{aligned}
& T P R=\frac{T P}{T P+F N} \times 100 \\
& F P R=\frac{F P}{F P+T N} \times 100 \\
& \text { Precision }=\frac{T P}{T P \_F P} \times 100 \\
& \text { Accuracy }=\frac{F P+T N}{T P+F P+T N+F N} \times 100
\end{aligned}
$$

where $T P, F P, T N, F N$ denote true positive, false positive, true negative, and false negative, respectively. We also implement method in [16] that is most like our proposed approach. Table 2 shows the comparative performance result.

Table 2. Comparative performance result

\begin{tabular}{ccc}
\hline Measure & Previous [16] & Proposed \\
\hline TPR & $96.45 \%$ & $98.65 \%$ \\
FPR & $3.24 \%$ & $3.11 \%$ \\
Precision & $55.34 \%$ & $58.19 \%$ \\
Accuracy & $96.83 \%$ & $97.02 \%$ \\
\hline
\end{tabular}

The proposed method is better in $T P R$ and precision performance measure and slightly better but not statistically significant in accuracy and FPR. This means that the proposed approach is more like human expert's decision especially in true positive area. Figure 7 demonstrate human expert's decision, previous result, and the proposed method's result, respectively.

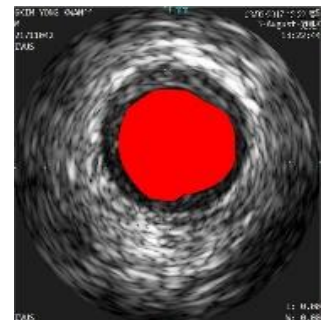

(a)

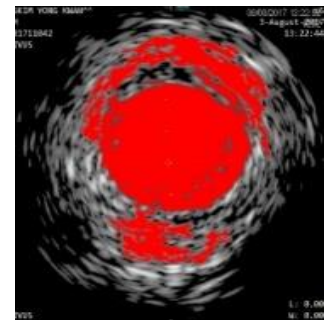

(b)

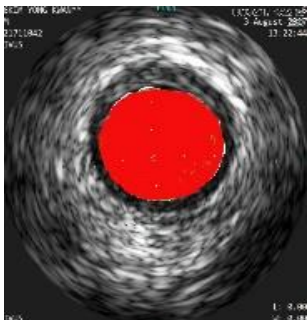

(c)

Figure 7. Improved case ibn performance; (a) human, (b) previous [16], (c) proposed

\section{CONCLUSION}

Although medical image segmentation is an established and mature field, fully automatic segmentation of medical image data remains an unsolved problem due to the size of the dataset, the complexity of anatomical shapes, overlap of gray level values between neighboring tissues and organs, lack of consistently distinct boundaries and sampling artifacts and noise. Making fully automatic segmentation of intima/adventitia area from IVUS that contains many types of artifacts in the image is much necessary attempt that can get rid of operator subjectivity in the analysis. In this paper, we propose a fully automatic segmentation method using Bezier curve in adventitia/intima boundary interpolation. To successfully reconstruct a surface model from contours, it is important to accurately identify and extract features of interest from medical images and Bezier curve interpolation is a feasible approach as we demonstrate in this paper. 
In experiment with 800 vessel IVUS images, the proposed method shows as good as the best result of reported FCM clustering approach in overall discrepancy from human expert's manual measurement where $84.4 \%$ cases have less than $25 \mathrm{~mm} 2$ discrepancy. We take further curve fitting analysis and the proposed method is better than or as good as the previous most similar approach in all performance indices in direct comparison. The proposed method showed more overestimation of the area between adventitia and Intima than underestimation in the post-hoc analysis thus more conservative treatment is needed for the future research.

\section{REFERENCES}

[1] T. Ma, B. Zhou, T. K. Hsiai, and L. L. Shung, "A review of intravascular ultrasound-based multimodal intravascular imaging: the synergistic approach to characterizing vulnerable plaques," Ultrasonic imaging, vol. 38, no. 5, pp. 314-331, 2016.

[2] M. Faraji, I. Cheng, I. Naudin, and A. Basu, "Segmentation of arterial walls in intravascular ultrasound crosssectional images using extremal region selection," Ultrasonics, vol. 84, pp. 356-365, 2018.

[3] A. Katouzian, E. A. D. Angelini, S. G. Carlier, J. S. Suri, N. Navab N, and A. F. Laine, "A state-of-the-art review on segmentation algorithms in intravascular ultrasound (IVUS) images," IEEE Transactions on Information Technology in Biomedicine, vol. 16, no. 5, pp. 823-834, 2012.

[4] M. Eslamizadeh, G. Attarodi, N. J. Dabanloo, J. F. Sedehi, and S. K. Setaredan, "The Segmentation of Lumen Boundaries at Intravascular Ultrasound Images Using Fuzzy Approach,” 2017 Computing in Cardiology (CinC), Rennes, 2017, pp. 1-4.

[5] T. Araki, S. K. Banchhor, N. D. Londhe, N. Ikeda, P. Radeva, D. Shukla, L. Saba, A. Balestrieri, A. Nicolaides, S. Shafique, J. R. Laird, and J. S. Suri, "Reliable and accurate calcium volume measurement in coronary artery using intravascular ultrasound videos," Journal of medical systems, vol. 40, no. 3, 2016.

[6] A. Taki, Z. Najafi, A. Roodaki, S. K. Setarehdan, R. A. Zoroofi, A. Konig, and N. Navab, "Automatic segmentation of calcified plaques and vessel borders in ivus images," International Journal of Computer Assisted Radiology and Surgery, vol 3, no. 3-4, pp. 347-354, 2008.

[7] X. Zhu, P. Zhang, J. Shao, Y. Cheng, Y. Zhang, and J. Bai, "A snake-based method for segmentation of intravascular ultrasound images and its in vivo validation," Ultrasonics, vol. 51, no. 2, pp. 181-189, 2011.

[8] E. G. Mendizabal-Ruiz, M. Rivera, and I. A. Kakadiaris, "Segmentation of the luminal border in intravascular ultrasound b-mode images using a probabilistic approach," Medical image analysis, vol. 17, no. 6, pp. 649-670, 2013.

[9] D. M. Herrington, T. Johnson, P. Santago, and W. E. Snyder, "Semi-automated boundary detection for intravascular ultrasound," Proceedings Computers in Cardiology, Durham, NC, USA, 1992, pp. 103-106.

[10] M. Sonka, X. Zhang, M. Siebes, M. S. Bissing, S. C. Dejong, S. M. Collins, and C. R. McKay, "Segmentation of intravascular ultrasound images: a knowledge-based approach," IEEE Transactions on Medical Imaging, vol. 14, no. 4, pp. 719-732, 1995.

[11] K. M. Meiburger, U. R. Acharya, and F. Molinari, "Automated localization and segmentation techniques for Bmode ultrasound images: A review," Computers in biology and medicine, vol. 92, pp. 210-235, 2018.

[12] S. Su, Z. Hu, Q. Lin, W. K. Hau, Z. Gao, and H. Zhang, "An artificial neural network method for lumen and mediaadventitia border detection in IVUS," Computerized Medical Imaging and Graphics, vol. 57, pp. 29-39, 2017.

[13] L. Lo Vercio, J. L. Orlando, M. del Fresno, and I. Larrabide, "Assessment of image features for vessel wall segmentation in intravascular ultrasound images," International journal of computer assisted radiology and surgery, vol. 11, no. 8, pp. 1397-1407, 2016.

[14] J. Yan, D. Lv, and Y. Cui, "A novel segmentation approach for intravascular ultrasound images," Journal of Medical and Biological Engineering, vol. 37, no. 3, pp. 386-394, 2017.

[15] H. Lazrag, K. Aloui, and M. S. Naceur, "Automatic segmentation of lumen in intravascular ultrasound images using fuzzy clustering and active contours," International Conference on Control, Engineering \& Information Technology, vol. 1, pp. 58-63, 2013.

[16] S. M. Dehnavi, M. P. Babu, M. Yazchi, and M. Basij, "Automatic soft and hard plaque detection in IVUS images: A textural approach," 2013 IEEE Conference on Information and Communication Technologies, Thuckalay, Tamil Nadu, India, 2013, pp. 214-219.

[17] K. B. Kim, D. H. Song, and S. S. Yun, "Automatic Extraction of Blood Flow Area in Brachial Artery for Suspicious Hypertension Patients from Color Doppler Sonography with Fuzzy C-Means Clustering," Journal of information and communication convergence engineering, vol. 16, no. 4, pp. 258-263, 2018.

[18] H. J. Park and K. B. Kim, "Morphological Detection of Carotid Intima-Media Region for Fully Automated Thickness Measurement by Ultrasonogram," Journal of information and communication convergence engineering, vol. 15 , no. 4 , pp. $250-255,2018$.

[19] H. J. Park and K. B. Kim, "Extracting acoustic shadowing from ultrasound image using local difference," Indonesian Journal of Electrical Engineering and Computer Science (IJEECS), vol. 13, no. 1, pp. 205-209, 2019.

[20] K. B. Kim, J. H. Lee, H. J. Park, and D. H. Song, "Auto-segmentation of Intima/Adventitia of the Vessel by Subtracting Different types of Binarization Results," Proceedings of International conference on future information \& communication engineering, pp. 108-111, 2019.

[21] K. I. Dimitris and G. Papamichalis, "Automatic Segmentation of the Lung Fields in Portable Chest Radiographs based on Bezier Interpolation of Salient Control Points," 2008 IEEE International Workshop on Imaging Systems and Techniques, Crete, 2008, pp. 82-87. 
[22] R. T. Farouki, "The Bernstein polynomials basis: a centennial retrospective," Computer Aided Geometric Design, vol. 29, no. 6, pp. 379-419, 2012.

[23] A. Foroozandeh, A. Kerayechiyani, M. Gachpazan, M. Momennezhad, and S. Nasseri, "3D Reconstruction Using cubic Bezier spline curves and active contours," Iranian Journal of Medical Physics, vol. 9, no. 3, pp. 169-176, 2012.

[24] H. S. Gan, T. T. Swee, and A. H. A. Karim, "Medical Image Visual Appearance Improvement Using Bihistogram Bezier Curve Contrast Enhancement: Data from the Osteoarthritis Initiative," Scientific World Journal, vol. 2014, 2014.

[25] Z. Tian, X. Si, Y. Zheng, Z. Chen, and X. Li, "Multi step medical image segmentation based on reinforcement learning," Journal of Ambient Intelligence and Humanized Computing, 2020.

[26] B. Simsek and A. Yardimci, "Using Bezier curves in medical applications," Filomat, vol. 30, no. 4, pp. 937-943, 2016.

[27] J. O. Park, B. C. Ko, H. J. Park, J. Y. Nam, "Automatic Identification of the Lumen Border in Intravascular Ultrasound Images," The Korea Inormation Processing Spciety Journal Transactions B, vol. 19B, no. 3, pp. 201-208, 2012.

\section{BIOGRAPHIES OF AUTHORS}
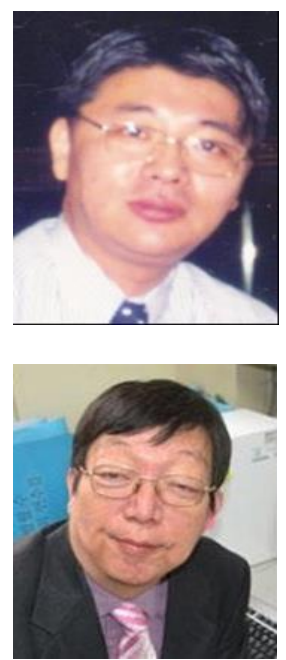

Kwang Baek Kim received his M.S. and Ph.D. degrees from the Department of Computer Science, Pusan National University, Busan, Korea, in 1993 and 1999, respectively. From 1997 to the present, he is a professor at the Division of Computer and Information Engineering, Silla University, Korea. He is currently an associate editor for Journal of Intelligence and Information Systems and The Journal of Information and Communication Convergence Engineering. His research interests include artificial intelligence, fuzzy clustering, data mining, image processing, and bioinformatics. He is currently the chief editor of Journal of Information and Communication Convergence Engineering.

Doo Heon Song received his B.S. from Seoul National University in 1981 and M.S. from the Korea Advanced Institute of Science and Technology in 1983 in Computer Science. He received his Ph.D. candidate Certificate in Computer Science from the University of California at Irvine in 1994. He has been a professor at Department of Computer Games, Yong-in Songdam College, Korea, since 1997. His research interests include artificial intelligence, image processing, and computer game design. He is currently the associate editor of Journal of Information and Communication Convergence Engineering. 